\title{
A THEOREM ON INFINITE POSITIVE MATRICES ${ }^{1}$
}

\section{E. NETANYAHU AND M. REICHAW}

1. Let $A=\left(a_{i j}\right)$ be an infinite matrix with positive elements $a_{i j}>0, i, j=0,1, \cdots$, (matrices $\left(a_{i j}\right)$ with $a_{i j}>0$ will be called in the sequel positive matrices).

It was proved in [3], that

(1) if $A$ is a finite positive matrix, a unique doubly stochastic matrix $T$ exists such that $T=D_{1} A D_{2}$ where $D_{1}$ and $D_{2}$ are diagonal matrices with all elements on the diagonal positive and are unique up to a scalar factor.

The method used in [3], and introduced first in [4], is a constructive one and consists in alternate normalizing rows and columns of $A$ and proving the convergence of this procedure. Another proof of (1) was given in [1]. This second proof uses besides Brouwer's fixed point theorem the fact, that

(2) the set $\left\{x=\left(x_{0}, x_{1}, \cdots, x_{n}\right) ; x_{i}\right.$ real numbers, $\sum_{i=0}^{n} x_{i}^{2}=1$ and $\left.x_{i} \geqq 0\right\}$ is homeomorphic to an $n$-dimensional ball.

Although a purely existential one, this second proof contains a statement about the existence of directions of fixed points for some mapping defined by help of a finite matrix $A$. In this paper we note that statement (1) does not hold for infinite matrices and prove a theorem generalizing properly (1) to the case of infinite matrices. Essentially, both proofs in [1] and in [3] could be, with some nontrivial changes, applied to give the desired generalization. The difficulty in generalizing the proof given in [3] consists i.a. in the fact that for an infinite matrix $\sum_{j} a_{i j}$ (or $\sum_{i} a_{i j}$ ) is not always finite. The idea of our proof is similar to that of [1] except that (2) is not used and that Brouwer's theorem is replaced by the theorem of Schauder (see [2]). In the sequel a matrix $A=\left(a_{i j}\right)$ with $a_{i j}>0$ will be called a positive matrix and a diagonal matrix with positive diagonal elements will be called a positive diagonal matrix. Finally $\delta_{i j}=0, i \neq j ; 1, i=j$, will denote the delta of Kronecker.

2. Before generalizing (1) to the case of infinite matrices let us note that

(3) if $A=\left(a_{i j}\right)$ is infinite with $a_{i j}=1$ for $i, j=0,1,2, \ldots$ then positive diagonal matrices $D_{1}$ and $D_{2}$ for which $T=D_{1} A D_{2}$ is doubly stochastic do not exist.

Received by the editors September 11, 1967.

1 This research was supported by the U. S. Air Force Grant AF-AFOSR 396-63, monitored by the Office of Scientific Research. 
Indeed, if $D_{1}=\left(\delta_{i j} p_{i}\right)$ and

$$
D_{2}=\left(\delta_{i j} q_{j}\right), \quad p_{i}>0, \quad q_{j}>0, \quad i, j=0,1,2, \cdots
$$

then for a doubly stochastic matrix $T=D_{1} A D_{2}$ one has $t_{i j}=p_{i} q_{j}$ and $p_{i}=p_{j}$ for all $i, j=0,1, \cdots$, which is impossible.

We show now, that

(4) if $A=\left(a_{i j}\right)$ with $a_{i j}=1$ for all $i, j=0,1, \cdots$ and if there exist positive diagonal matrices $D_{1}=\left(\delta_{i j} p_{i}\right)$ and $D_{2}=\left(\delta_{i j} q_{j}\right)$ such that for $T=D_{1} A D_{2}=\left(t_{i j}\right)$ one has $\sum_{j} t_{i j}=\alpha_{i}$ and $\sum_{i} t_{i j}=\beta_{j}, i, j=0,1, \cdots$, then $\sum_{i=0}^{\infty} \alpha_{i}=\sum_{0}^{\infty} \beta_{j}<\infty$.

Indeed, condition $\sum_{j=0}^{\infty} t_{0 j}=\alpha_{0}$ i.e. $p_{0} \sum_{0}^{\infty} q_{j}=\alpha_{0}$ implies $\sum_{0}^{\infty} q_{j}<\infty$ and similarly $\sum_{0}^{\infty} p_{i}<\infty$. But then $\left(\sum_{0}^{\infty} p_{i}\right)\left(\sum_{0}^{\infty} q_{j}\right)=\sum_{0}^{\infty} \alpha_{i}=\sum_{0}^{\infty}$ $\beta_{j}<\infty$.

Property (4) justifies the assumption $\sum_{0}^{\infty} \alpha_{i}=\sum_{0}^{\infty} \beta_{j}<\infty$ made in the following that

Theorem. Let $A=\left(a_{i j}\right)_{i, j=0,1}, \ldots$ be an infinite positive matrix such

(a) there exists a constant $M$ with $a_{i j} \leqq M$ and

(b) there exists a column (say the 0th column) and constants $L_{0}$ and $M_{0}$ such that for every $i, k=0,1, \cdots$ one has $a_{i 0} \leqq M_{0} a_{i k}$ and $a_{i k} \leqq L_{0} a_{i 0}$. Let further $\left\{\alpha_{i}\right\}$ and $\left\{\beta_{j}\right\}$ be sequences of positive numbers such that $\sum_{0}^{\infty} \alpha_{i}=\sum_{0}^{\infty} \beta_{j}<\infty$.

Then there exist positive diagonal matrices $D_{1}$ and $D_{2}$ such that for $T=D_{1} A D_{2}=\left(t_{i j}\right)$ one has

(c) $\sum_{j} t_{i j}=\alpha_{i}$ and $\sum_{i} t_{i j}=\beta_{j}, \quad i, j=0,1, \cdots$.

Proof. Putting $N_{0}=1$ and $N_{i}=N \geqq 1$ for $i \geqq 1$ and multiplying $A$ on the right by the matrix $D=\left(\delta_{i j} N_{i}\right)$ one can by choosing $N$ sufficiently large obtain by (b) that, for the matrix $B=A D=\left(b_{i j}\right)$,

(d) $b_{i 0} \leqq b_{i k}$ and $b_{i 0} \leqq\left(\beta_{0} / \sum_{k \geq 1} \beta_{k}\right) b_{i k}$ holds for every $i, k=0,1$, ..., $k \neq 0$.

It suffices obviously to find positive diagonal matrices $P$ and $Q$ such that $T=P B Q=\left(t_{i j}\right)$ satisfies (c) (then $D_{1}=P$ and $D_{2}=D Q$ ). Now consider the equations

(e $\left.u_{i}\right) \sum_{j} b_{i j} x_{j}^{\prime}=\alpha_{i}$,

(e $\left.e_{2}\right) x_{k} \sum_{i} b_{i k} u_{i}=\beta_{k}, i, k=0,1, \cdots$.

Expressing $x_{k}$ in terms of $x_{j}^{\prime}$ we get

(f) $x_{k}=\beta_{k} / f_{k}\left(\left\{x_{j}^{\prime}\right\}\right)$, where $f_{k}\left(\left\{x_{j}^{\prime}\right\}\right)=\sum_{i \geq 0}\left(\alpha_{i} b_{i k} / \sum_{j \geqq 0} b_{i j} x_{j}^{\prime}\right)$.

Evidently, if one finds a sequence $\left\{x_{j}^{\prime}\right\}$ with $x_{j}^{\prime}>0$ such that in (f) $x_{k}=x_{k}^{\prime}$ for every $k \geqq 0$, then calculating $u_{i}$ from $\left(\mathrm{e}_{1}\right)$ and putting $P=\left(\delta_{i j} u_{i}\right)$ and $Q=\left(\delta_{i j} x_{j}\right)$ we have the desired matrices $P$ and $Q$. In other words one looks for any fixed point $x=\left\{x_{k}\right\}_{k=0,1}, \ldots, x_{k}>0$ of the mapping defined by (f). To get such a fixed point let us denote 
$\xi_{k}=x_{k}^{\prime} / x_{0}^{\prime}$ and $\eta_{k}=x_{k} / x_{0}, k=1,2, \cdots$, (we call $\left\{\xi_{k}\right\}$ and $\left\{\eta_{k}\right\}$ "directions").

Then by (f) one has

$$
\eta_{k}=\frac{\beta_{k}}{\beta_{0}} \frac{g_{0}\left(\left\{\xi_{j}\right\}\right)}{g_{k}\left(\left\{\xi_{j}\right\}\right)}, \quad \text { where } \quad g_{k}\left(\left\{\xi_{j}\right\}\right)=\sum_{i \geq 0} \frac{\alpha_{i} b_{i k}}{b_{i 0}+\sum_{j \geq 1} b_{i j} \xi_{j}} .
$$

Let us confine ourselves to $\xi_{j} \geqq 0$ such that $\sum_{j \geqq 1} \xi_{j} \leqq 1$, i.e. such that the point $x=\left(\xi_{1}, \xi_{2}, \cdots\right)$ belongs to the intersection $C \cap S$ of the cone $C=\left\{x=\left(\xi_{1}, \xi_{2}, \cdots\right) ; \xi_{i} \geqq 0, x \in l\right\}$ in the Banach space $l$ with the unit ball $S$ of this space. ${ }^{2}$ Since $\sum \alpha_{i}<\infty$ we obtain by (a) that $\eta_{k}$ exists for all $k=1,2, \cdots$ and obviously by (b) $\eta_{k}>0$. By (d) it follows that $\eta_{k} \leqq \beta_{k} / \beta_{0}$ and that $\sum_{k=1}^{\infty} \eta_{k} \leqq 1$.

Thus, by $\sum \beta_{k}<\infty$, formula (g) defines a continuous mapping $F$ of $C \cap S$ into a compact subset of $C \cap S$. By the fixed point theorem of Schauder (see [2]) there exists a point $\bar{x}=\left(\bar{\xi}_{1}, \bar{\xi}_{2}, \cdots\right)$ such that $F(\bar{x})=\bar{x}$. This point is an invariant direction of the mapping $F$. Take as in [1] any point $\left(x_{0}^{\prime}, x_{1}^{\prime}, \cdots\right)$ on this direction with $x_{0}^{\prime}>0$. Then $x_{j}=\theta x_{j}^{\prime}, j=0,1, \cdots$, and putting $x_{j}^{\prime}$ into $\left(\mathrm{e}_{1}\right)$ we obtain the sequence $\left\{u_{i}\right\}_{i=0,1}, \ldots$ Then by $\left(\mathrm{e}_{1}\right)$ and $\left(\mathrm{e}_{2}\right)$ we have

$$
\sum_{i} u_{i} \sum_{j} b_{i j} x_{j}^{\prime}=\sum \alpha_{i}=\sum \beta_{i}=\theta \sum u_{i} \sum_{j} b_{i j} x_{j}^{\prime} .
$$

Thus $\theta=1$ and the sequences $\left\{u_{i}\right\}$ and $\left\{x_{j}\right\}$ satisfy both $\left(e_{1}\right)$ and $\left(e_{2}\right)$.

The theorem is proved.

REMARKs. Let us note that in case of a finite positive matrix $A=\left(a_{i j}\right)$ all the assumptions of the Theorem hold. Finally let us note that if $A=\left(a_{i j}\right)_{i, j=0,1}, \ldots$ is infinite and $a_{i j}=1 / 2^{j+1}, i, j=0,1,2, \cdots$, then $A$ is obviously a stochastic matrix but the argument applied in (3) shows that positive diagonal matrices $D_{1}$ and $D_{2}$ for which $T$ $=D_{1} A D_{2}$ is doubly stochastic do not exist.

\section{REFERENCES}

1. M. V. Menon, Reduction of a matrix with positive elements to a doubly stochastic matrix, Proc. Amer. Math. Soc. 18 (1967), 244-247.

2. J. Schauder, Der Fixpunktsatz in Funktionalräumen, Studia Math. 21 (1930), 171-180.

3. R. Sinkhorn, $A$ relationship between arbitrary positive matrices and doubly stochastic matrices, Ann. Math. Statist. 35 (1964), 876-879.

N. J.

4. L. Welch, Unpublished report of the Institute of Defense Analysis, Princeton,

Technion Haifa, IsRael and Cornell University

${ }^{2} l$ denotes the Banach space of all sequences $x=\left(\xi_{1}, \xi_{2}, \cdots\right)$ with $\xi_{i}$ real and $\sum\left|\xi_{i}\right|<\infty$. 